\title{
Agomelatine in the treatment of mild-to-moderate depression in patients with cardiovascular disease: results of the national multicenter observational
} study PULSE

\author{
This article was published in the following Dove Press journal: \\ Neuropsychiatric Disease and Treatment \\ 21 April 2017 \\ Number of times this article has been viewed
}

\author{
Vladimir E Medvedev \\ Department of Psychiatry, \\ Psychotherapy and Psychosomatic \\ Pathology, RUDN University, \\ Moscow, Russia
}

Correspondence: Vladimir E Medvedev Department of Psychiatry, Psychotherapy and Psychosomatic Pathology, RUDN University, Slavyansky bulvar, I-95,

Moscow, 121352 Russia

$\mathrm{Tel} / \mathrm{fax}+74953502875$

Email medvedev_ve@pfur.ru
Background: PULSE was a large, observational, multicenter study designed to evaluate the efficacy and safety of agomelatine in the treatment of major depression in patients with cardiovascular disease (CVD).

Methods: Patients with mild-to-moderate major depressive episodes, without psychotic symptoms, were treated as outpatients or in cardiac facilities in 46 regions of Russia. The patients received antidepressant monotherapy with agomelatine 25 or $50 \mathrm{mg}$, once daily, for 12 weeks. Results: The mean age of the patients $(\mathrm{N}=896)$ was $51.4 \pm 9.9$ years, and $68.5 \%$ were women. A progressive improvement in the total score on both the anxiety and depression subscales of the Hospital Anxiety and Depression Scale (HADS), from 13.1 \pm 3.8 and 13.9 \pm 3.1 at baseline to $3.7 \pm 2.8$ and $3.9 \pm 3.0$, respectively, was observed by 12 weeks. All individual HADS scores improved rapidly; the change between visits was also significant $(P<0.0001)$. The majority $(84.6 \%)$ were remitters (HADS total score $<7$ ) by 12 weeks. The Clinical Global Impression - Severity and Improvement scores also improved quickly. The mean hypochondria index (Whiteley Index) decreased significantly from $48.0 \pm 11.8$ at baseline to $25.2 \pm 9.2$ at 12 weeks $(P<0.0001)$. The main hemodynamic indices improved or remained stable, and biochemical parameters reflecting liver function (aspartate aminotransferase, alanine aminotransferase, gamma-glutamyl transpeptidase, alkaline phosphatase, total bilirubin) did not exceed three times the upper limits of established norms.

Conclusion: Agomelatine resulted in statistically significant improvements in depressive symptoms, anxiety, and hypochondria in depressed patients with CVD, and had good tolerability. Our data suggest that agomelatine is safe to treat depression in patients with CVD.

Keywords: depression, cardiovascular disease, agomelatine, anxiety, hypochondria, tolerability

\section{Introduction}

Patients with cardiovascular disease (CVD) are three times more likely to experience depression than other members of the community. ${ }^{1}$ Depression is more frequently observed in ambulatory cardiac patients $(9.3 \%)$ than in the general population $(4.8 \%)$, and in patients undergoing treatment in hospital. ${ }^{2,3}$ Large, international, epidemiologic studies have shown that many patients treated by physicians, including cardiologists and neurologists, have some form of clinical depression that requires antidepressant therapy. ${ }^{4}$ Depression affects around one-fifth of heart failure patients $(21 \%)$ and between $15 \%$ and $20 \%$ of patients admitted to hospital for myocardial infarction. ${ }^{5,6}$ Patients with CVD diagnosed with depression have an increased risk of poor cardiovascular 
outcomes. ${ }^{7,8}$ However, it is not clear whether treatment with antidepressants improves or worsens this risk. ${ }^{9,10}$

Tricyclic antidepressants and monoamine oxidase inhibitors are contraindicated in many patients with CVD as they are considered cardiotoxic, while selective serotonin reuptake inhibitors (SSRIs) are considered effective for treating depression in CVD patients and may even improve patient prognosis. ${ }^{11,12}$ However, the SADHARTCHF (Sertraline Against Depression and Heart Disease in Chronic Heart Failure) study showed that while sertraline was safe, it was not effective in treating depression and had no impact on short- or long-term cardiovascular events or survival. ${ }^{13}$ Examination of data from the REGARDS (Reasons for Geographic and Racial Differences in Stroke) study in stroke patients using an antidepressant at baseline found antidepressant use was associated with a small increase in risk of all-cause mortality. ${ }^{14}$ Furthermore, antidepressants have been associated with an increased risk of stroke in two other studies. ${ }^{15,16}$ Nonetheless, a large cohort study found no association between SSRI use and poor cardiovascular outcomes, and even reported a reduction in risk of myocardial infarction. ${ }^{17}$

There is a real need for an effective and safe agent for the treatment of depression in patients with CVD. It is estimated that approximately $15 \%-25 \%$ of depressed cardiac patients stop taking antidepressants due to adverse events within 6 months of treatment initiation, highlighting the importance of selecting an antidepressant with good tolerability. ${ }^{18}$ Treatment of depression in CVD patients requires careful monitoring, and drugs that are known to be well tolerated and safe should be the first choice of the prescriber.

Agomelatine has been shown to demonstrate antidepressant efficacy in both short- and long-term studies ${ }^{19,20}$ as well as in clinical practice, ${ }^{21-23}$ and is unique among antidepressive agents in its ability to relieve anhedonia early in treatment. ${ }^{24,25}$ Due to its novel pharmacology (melatonergic receptor agonist and 5-hydroxytryptamine $2 \mathrm{C}$ [5HT2C] receptor antagonist) and good tolerability profile, ${ }^{19,26}$ agomelatine could be a good candidate for the treatment of depression in patients with CVD, but no specific studies have evaluated agomelatine in CVD patients so far. An increase in liver transaminases has been observed in some patients treated with agomelatine, and hence, liver function tests are required. The purpose of this national, multicenter, observational program "PULSE" was therefore to obtain data on the efficacy and tolerability of agomelatine at standard doses for the treatment of mild and moderate depressive disorders in a range of patients with CVD in attending cardiologists in Russia.

\section{Patients and methods}

This study included men and women with CVD, between 18 and 65 years old, having a score of $\geq 11$ points on the depression subscale of the Hospital Anxiety and Depression Scale (HADS), and fulfilling the criteria of the International Classification of Diseases, Tenth Edition, for depressive episodes of mild or moderate severity without psychotic symptoms, suicidal thoughts and intentions, and seasonal changes of state.

For women with intact reproductive function, an obligatory condition of participation was the use of adequate medical contraception. This study was approved by the Local Ethics Committee of RUDN University. All patients provided written informed consent to participate in the study. The patients were diagnosed with depression primarily by cardiologists. This diagnosis was confirmed by a psychiatrist. Antidepressive treatment was prescribed by cardiologists.

Patients who were under psychiatrist supervision and/or were taking any psychotropic drugs, who had alcoholism or a history of drug addiction, a history of idiosyncrasy, or had been previously treated unsatisfactorily with agomelatine (administered at an adequate dose [25-50 mg/day] and for adequate duration [at least 4 weeks]) were not included. Patients were also excluded if they had severe somatic disorders including oncologic, hepatologic, and neurologic diseases, were taking inhibitors of cytochrome P450 1A2 (eg, ciprofloxacin, fluvoxamine), were pregnant or breastfeeding, or had lactase deficiency, galactosemia, or glucosegalactose malabsorption.

Patients received a once-daily treatment of agomelatine for depression, at either $25 \mathrm{mg}$ or $50 \mathrm{mg}$ always at bedtime, over a period of 12 weeks. During this time, patients were examined by a doctor at four mandatory visits: enrollment visit (W0), and after 3 weeks (W3), 6 weeks (W6), and 12 weeks (W12). The following range of psychometric instruments was used for patient assessment at each visit: HADS questionnaire, Clinical Global Impression - Severity (CGI-S) and Clinical Global Impression - Improvement (CGI-I) scales, a visual analog scale (VAS), reactive anxiety subscale of Spielberger-Khanin anxiety scale, Whitely hypochondria index, and Short Form 36 (SF-36) quality-of-life questionnaire.

Treatment tolerability was assessed by the spontaneous reporting of complaints by patients and by changes in the main clinical parameters. A standardized, routine physical examination included assessment of blood pressure, heart rate, body weight, and biochemistry at each visit. Liver enzyme monitoring (aspartate aminotransferase 
and alanine aminotransferase) was performed following the recommendations of the Agomelatine Summary of Product Characteristics including the following: performing baseline liver function tests in every patient before starting treatment with agomelatine, not starting treatment if serum transaminases exceeded three times the upper limit of normal (ULN), and monitoring liver function at 3, 6, and 12 weeks.

Results were statistically processed using specialized computer programs (SPSS 16.0). Student's $t$-test for paired samples was used for within-group comparisons of continuous variables that were normally distributed, and Wilcoxon's test for those that were not normally distributed. Student's $t$-test for independent samples was used for between-group comparisons of continuous variables, and the Mann-Whitney test for those not normally distributed. The $\chi^{2}$ test was used for categorical variables. All tests were two-sided, and the type I error rate was $5 \%$.

\section{Results}

\section{Baseline data}

A total of 293 cardiologists from 46 regions in Russia enrolled 896 patients between October 2012 and April 2013. Of these, 20 patients (2.2\%) dropped out prematurely, leaving 876 patients eligible for analyses. The sociodemographic characteristics of the patients are presented in Table 1. Depression of mild or moderate severity occurred predominantly in women aged around 50 years (51.4 \pm 9.9 years) who were married, employed, and had a level of higher education (Table 1). In addition to CVD, patients typically had concomitant disorders affecting the endocrine, digestive, respiratory, and nervous systems (Table 2). A list of the most prescribed non-psychotropic therapy, administered to $>5 \%$ of patients, is presented in Table 3. Just under two-thirds of patients $(60 \%)$ were diagnosed with depression of moderate severity, with the remainder having a diagnosis of mild depressive episode.

The most prevalent symptoms of depression - diagnosed by cardiologists - in addition to depressed mood, included sleep disturbances, fatigue, cognitive impairment, and anhedonia (Figure 1). Cardiologists were less likely to identify ideas of insignificance, decreased libido, and changes in appetite.

\section{Effectiveness of therapy}

The majority of patients (758 [86.5\%]) included in the analyses were receiving agomelatine as a $25 \mathrm{mg}$ daily dose, with only 118 patients (13.5\%) receiving $50 \mathrm{mg}$ per day. The HADS total score was significantly reduced by 12 weeks and at each visit compared with the previous $(P<0.0001$,

Table I Baseline demographics and patient characteristics

\begin{tabular}{|c|c|c|c|}
\hline Parameter & Characteristic & $N(896)$ & $\%$ \\
\hline \multirow[t]{2}{*}{ Gender } & Male & 282 & 31.5 \\
\hline & Female & 614 & 68.5 \\
\hline Age (years; mean $\pm S D$ & $<30$ & 34 & 3.8 \\
\hline \multirow[t]{4}{*}{ [range] $=5 \mathrm{I} .4 \pm 9.9[2 \mathrm{I}-69])$} & $30-39$ & 89 & 9.9 \\
\hline & $40-49$ & 197 & 22 \\
\hline & $50-59$ & 347 & 38.7 \\
\hline & $\geq 60$ & 229 & 25.6 \\
\hline \multirow[t]{5}{*}{ Educational level } & Incomplete secondary education & 15 & 1.7 \\
\hline & Secondary education & 65 & 7.3 \\
\hline & Specialized secondary education & 258 & 28.8 \\
\hline & Undergraduate education & 40 & 4.5 \\
\hline & Higher education & 518 & 57.8 \\
\hline \multirow[t]{4}{*}{ Marital status } & Married & 561 & 62.6 \\
\hline & Divorced & 136 & 15.2 \\
\hline & Single & 79 & 8.8 \\
\hline & Widow & 120 & 13.4 \\
\hline \multirow[t]{6}{*}{ Professional activity/student } & Student & 6 & 0.7 \\
\hline & Presently working & 554 & 61.8 \\
\hline & Unemployed & 16 & 1.8 \\
\hline & Housewife & 43 & 4.8 \\
\hline & Retired & 191 & 21.3 \\
\hline & Disabled & 86 & 9.6 \\
\hline \multirow[t]{3}{*}{ Diagnosis (according to ICD-I0) } & F32.0 (single depressive episode, mild) & 375 & 41.8 \\
\hline & F32.I (single depressive episode, moderate) & 478 & 53.4 \\
\hline & F32 (not specified) & 43 & 4.8 \\
\hline
\end{tabular}

Abbreviations: SD, standard deviation; ICD-10, International Classification of Diseases, Tenth Edition. 
Table 2 Concomitant diseases

\begin{tabular}{llll}
\hline Organ system & Disease & N (896) & \% \\
\hline Cardiovascular system & Arterial hypertension & 775 & 264 \\
& Angina & 143 & 29.5 \\
& History of myocardial infarction & 164 & 16 \\
& Atherosclerotic cardiosclerosis & 211 & 18.3 \\
& Rhythm and conduction disturbances & 158 & 23.6 \\
Endocrine & Chronic heart failure & 118 & 17.6 \\
Digestive & Diabetes mellitus & 93 & 13.2 \\
Respiratory & Chronic gastroduodenitis & 41 & 10.4 \\
Nervous & Chronic obstructive bronchopulmonary disease & 243 & 4.6 \\
& Encephalopathy & 183 & 27.1 \\
& Tension headache & 46 & 20.4 \\
& Moderate cognitive impairment & 71 & 5.1 \\
& Migraine & 25 & 7.9 \\
& History of stroke & 144 & 2.8 \\
& Vegetative dystonia syndrome & 521 & 16.1 \\
\hline
\end{tabular}

Note: Concomitant diseases diagnosed in $>1 \%$ of patients.

Table 4). The majority of patients $(84.6 \%)$ were in remission by week 12 (HADS total score $<7$ ).

There was a statistically significant decrease in anxiety (Spielberger-Khanin questionnaire) at each subsequent visit compared with the previous visit, with the number of patients with severe reactive anxiety decreasing from $840(95.9 \%)$ to $131(15 \%)$ by week $12(P<0.0001)$ (Figure 2$)$. The Whiteley Index for hypochondria also decreased significantly $(P<0.0001)$ over the course of 12 weeks of treatment with agomelatine, with the number of patients with severe hypochondria decreasing from $784(89.5 \%)$ to $176(20.1 \%)$ (Figure 3).

Physicians reported an increase in the overall effectiveness of agomelatine as measured using the CGI-I scale, with $69.3 \%$ of patients classed as showing a "significant improvement" compared with baseline. The overall assessment of

Table 3 Main non-psychotropic therapy use

\begin{tabular}{lll}
\hline Drug & Patients $\mathbf{( N = 8 9 6 )}$ & Patients (\%) \\
\hline Perindopril & 369 & 41.18 \\
Aspirin & 339 & 37.83 \\
Bisoprolol & 261 & 29.13 \\
Indapamide & 240 & 26.79 \\
Atorvastatin & 175 & 19.53 \\
Amlodipine & 152 & 16.96 \\
Metoprolol & 84 & 9.38 \\
Rosuvastatin & 82 & 9.15 \\
Trimetazidine & 76 & 8.48 \\
Losartan & 57 & 6.36 \\
Valsartan & 50 & 5.58 \\
Clopidogrel & 49 & 5.47 \\
Hypothiazid & 47 & 5.25 \\
\hline
\end{tabular}

the severity of the patient's condition on the CGI-S scale also showed a steady increase in the proportion of patients in remission or "borderline state" (Figure 4). Furthermore, by the end of therapy, the number of patients graded as "moderately" and "substantially ill" had significantly reduced from $92.9 \%$ to $20.9 \%$.

The subjective assessment of patient health on the VAS improved significantly during 12 weeks of agomelatine treatment, dropping from 7.0 to 2.9 points $(P<0.0001)$. The improvements observed were also statistically significant compared with the previous visit $(P<0.0001)$.

In general, the assessment of agomelatine efficacy by cardiologists and patients was similar and was described as "good" to "excellent" by $96 \%$ of physicians and $97 \%$ of patients who completed the study.

\section{Quality of life}

Results from the SF-36 quality-of-life questionnaire indicated that agomelatine was highly efficacious and safe when treating depression in patients with CVD (Figure 5). Eight quality-of-life parameters allowed two integral parameters of the SF-36 scale to be characterized: physical and mental components of health. The values of both components increased early in the study and continued to increase until study completion with statistically significant improvements compared with baseline $(P<0.0001)$ (Figure 6).

\section{Tolerability}

The tolerability of agomelatine for the treatment of mild and moderate depression in patients with CVD was considered 


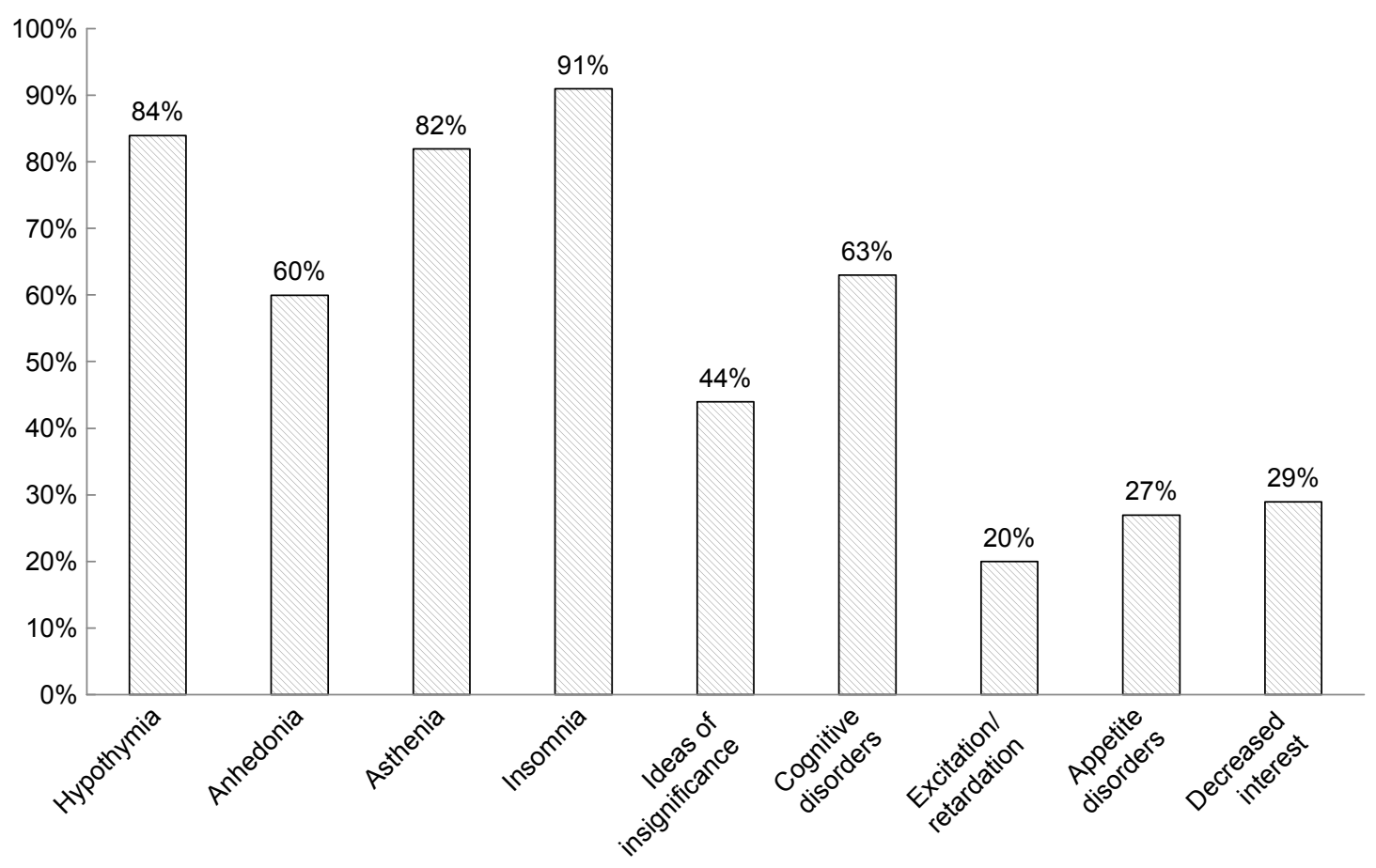

Figure I Frequency of distribution of depressive symptoms diagnosed by cardiologists.

good. Subjectively intolerable adverse events (increased anxiety and agitation, increased insomnia and dizziness) led to the withdrawal of only five out of the 20 patients who prematurely dropped out of the study. Treatment tolerability was considered as "excellent" by $82 \%$ of physicians and $75 \%$ of patients. The majority of patients $(83.6 \%)$ agreed to continue the treatment if necessary.

The physical characteristics of patients obtained at weeks 0 and 12 - before and after receiving the combined antidepressive and non-psychotropic (cardiotropic) therapy are summarized in Table 5. Patients' heart rate as well as systolic and diastolic blood pressure values were significantly lower at 12 weeks $(P<0.0001)$. Patient body weight after 12 weeks of therapy was lower than before therapy $(P<0.0001)$, but the difference did not exceed $5 \%$ of body weight at baseline.

Table 4 HADS-A and HADS-D scores over the 12-week treatment period

\begin{tabular}{lll}
\hline Weeks & HADS-A & HADS-D \\
\hline 0 & $13.1 \pm 3.8(\mathrm{I} 3)$ & $13.9 \pm 3.1(\mathrm{I} 3)$ \\
3 & $9.7 \pm 3.1(9)$ & $10.4 \pm 3.0(10)$ \\
6 & $6.3 \pm 2.9(6)$ & $6.9 \pm 2.9(7)$ \\
12 & $3.7 \pm 2.8(3)$ & $3.9 \pm 3.0(3)$
\end{tabular}

Notes: There were 876 patients included in this analysis. All values are presented as mean \pm standard deviation (median).

Abbreviations: HADS-A, Hospital Anxiety and Depression Scale - Anxiety: HADS-D, Hospital Anxiety and Depression Scale - Depression.
Liver function parameters obtained over the 12-week treatment period are summarized in Table 6. Transaminase levels were increased to below or equal to the ULN in around $3 \%-6 \%$ of patients after 6 weeks of treatment, but there were no cases where liver function parameters were threefold more than the ULN range at baseline. At baseline, $0.2 \%$ and $0.7 \%$ of patients, respectively, had values of alkaline phosphatase and gamma-glutamyl transpeptidase superior to normal, but less than or equal to three times the ULN. After treatment,

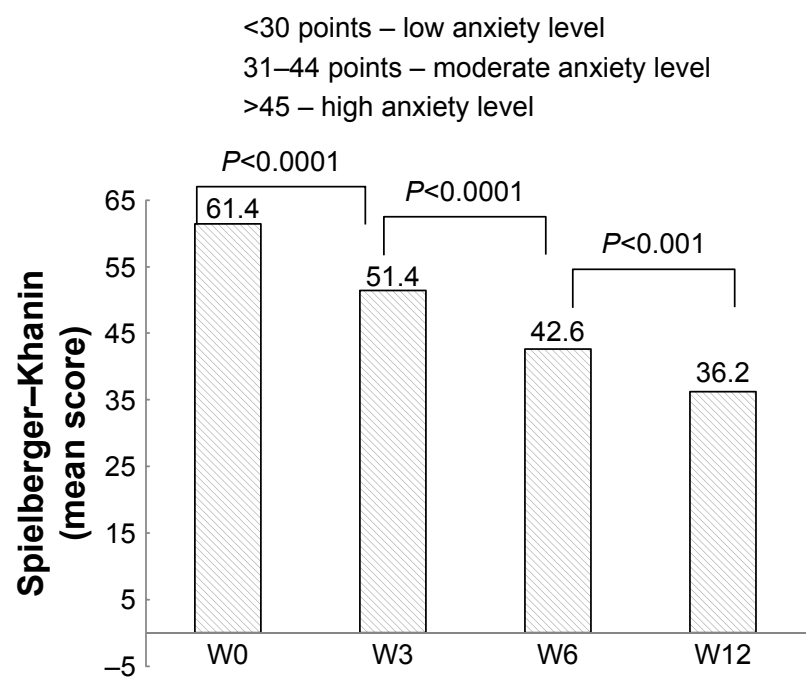

Figure 2 Mean Spielberger-Khanin reactive anxiety subscale scores from baseline to week (W) 12. 


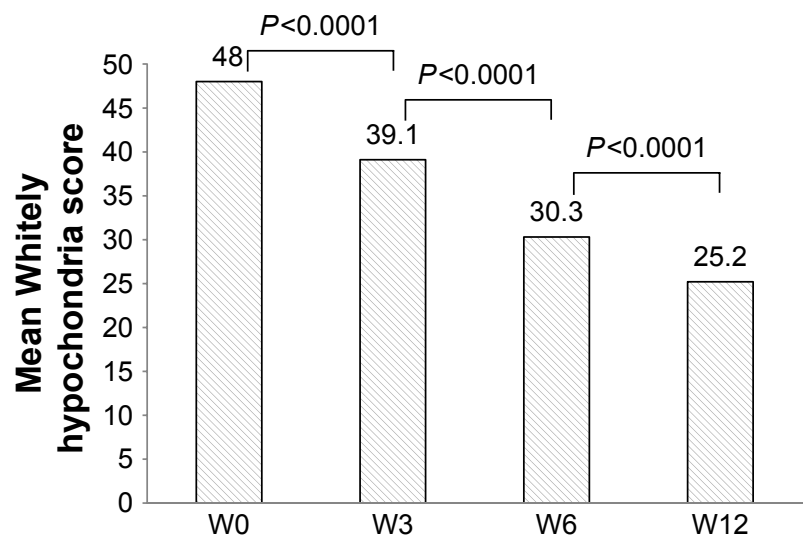

Figure 3 Mean Whiteley Index for hypochondria scores from baseline to week (W) 12.

no liver function parameters were threefold more than the ULN (Table 6).

\section{Discussion}

In this prospective, observational study, agomelatine proved effective for the treatment of depressive disorders in patients with CVD evaluated using a range of psychometric measures, and was well tolerated. Results were observed from the first post-baseline visit at week 3 and continued to improve throughout the study with statistically significant changes at each evaluation compared with the previous visit and with baseline. Early symptom relief is important, particularly in patients with CVD, as comorbid depression worsens CVD prognosis. ${ }^{27}$ Depression has also been associated with other behaviors that impact on CVD, such as medication nonadherence, that reduce the likelihood of successful disease management. ${ }^{27}$
An ideal treatment for depressive disorders would provide symptom relief followed by the restoration of normal functioning and prevention of relapse. In this study, HADS scores for anxiety and depression decreased from 13.1 and 13.9, respectively, at baseline to 3.7 and 3.9, respectively, at week 12 with agomelatine. Furthermore, the majority of patients $(84.6 \%)$ were in remission by week 12 . These results are in agreement with those obtained with agomelatine in clinical trials and clinical practice. The efficacy of agomelatine in patients with major depressive disorder has been extensively evaluated in a number of randomized, headto-head clinical trials. Long-term treatment with agomelatine demonstrates a sustained duration of action. ${ }^{28}$ A meta-analysis of published and unpublished short-term studies involving agomelatine in the treatment of depression that included 20 trials and around 7,500 participants suggested similar efficacy to standard antidepressants including paroxetine, fluoxetine, escitalopram, sertraline, and venlafaxine. ${ }^{19}$ Furthermore, those randomized to agomelatine were also less likely than those receiving comparator antidepressants to discontinue treatment because of adverse effects. A pooled analysis of four published 24-week studies suggested that treatment with agomelatine was at least as effective as treatment with SSRIs in the long term. ${ }^{29}$

CVD and depression often have overlapping symptoms such as fatigue, low energy, and difficulty in sleeping and carrying out daily routines of life. It is therefore not surprising that the symptoms of depression are sometimes thought to be due to CVD alone. Considering the prevalence of depression among CVD patients, it is important that cardiologists easily identify the key symptoms of depression such

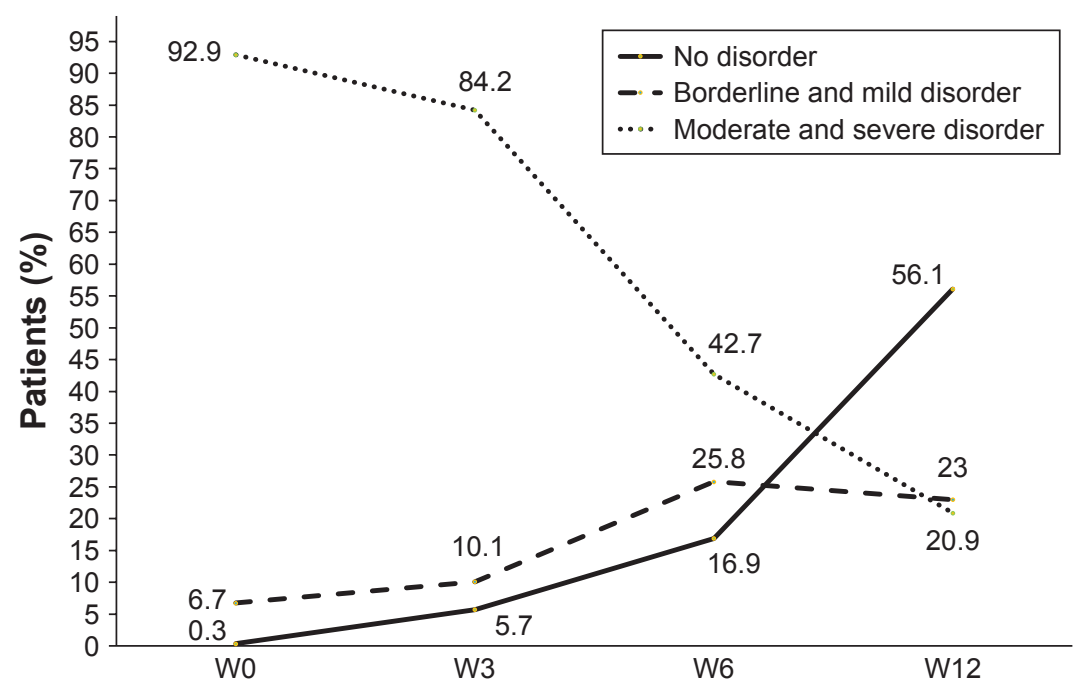

Figure 4 Proportion of patients with depression of varying severity from baseline to week (W) I2 (Clinical Global Impression - Severity). 


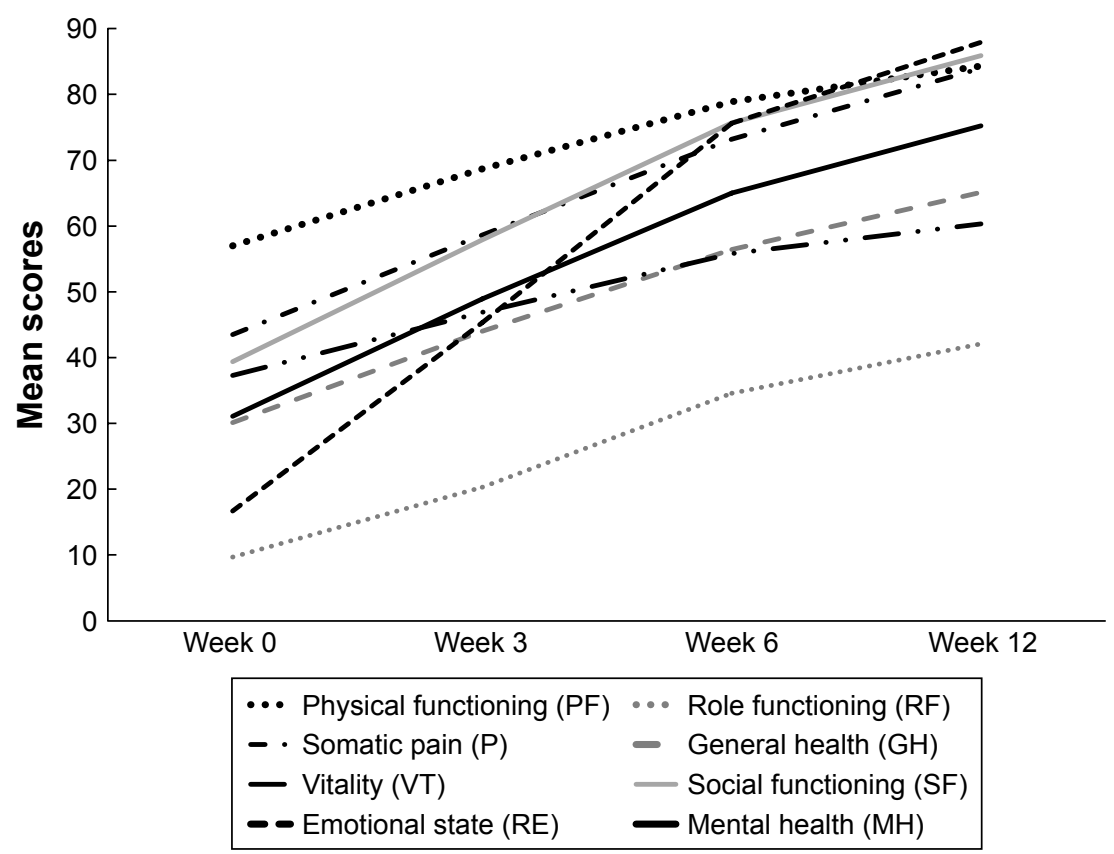

Figure 5 Mean scores for indicators of patients functioning according to the Short Form 36 quality-of-life questionnaire.

as hypothymia, anhedonia, fatigue, sleep disturbances, and cognitive impairments. One of the most common symptoms in this study was hypochondria, which could be caused by the presence of both serious somatic disease and affective (anxious-depressive) disorders, and was significantly reduced by the end of the study.

Agomelatine was also effective at relieving other key symptoms of depression including anxiety and hypochondria, both of which improved significantly at each visit compared with the last, throughout the study. Anxiety within depression is common and associated with worse prognosis, increased

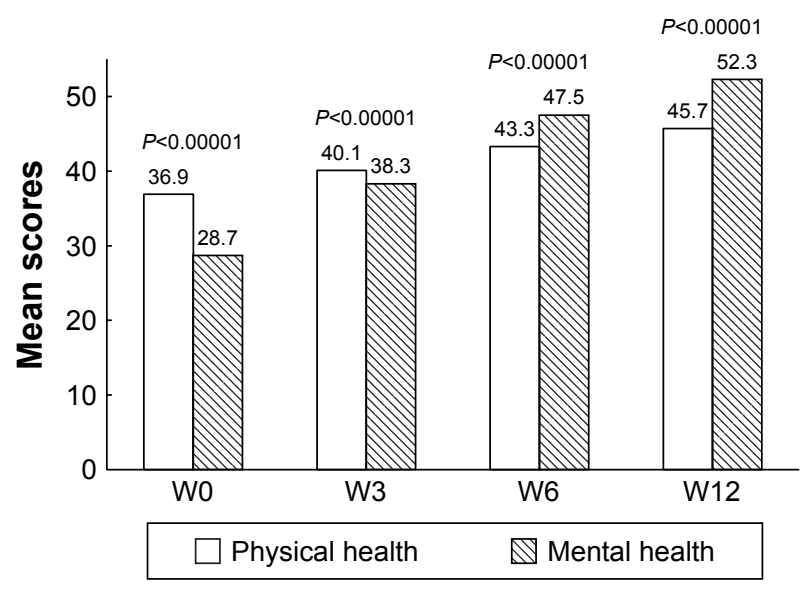

Figure 6 Mean scores for physical health and mental health components (Short Form 36 integrated indices of health) from baseline to week (W) 12 .

Note: $P$-values describe the change in physical and mental health scores between baseline and each visit. disability, and higher use of medication. The ability of agomelatine to improve anxiety in patients suffering from depression and CVD confirms findings from a pooled analysis of nearly 2,000 patients with major depressive disorder from six studies of 6-8 weeks duration: three placebo-controlled and three versus comparator antidepressants. ${ }^{30}$ In this analysis, agomelatine reduced the Hamilton Depression Rating Scale (HAM-D) score as early as the second week $(P<0.004)$ compared with placebo, and the reduction remained significant over the entire study $(P<0.001)$. Compared with the other drugs in the study, agomelatine proved more effective in reducing anxiety symptoms with a substantial difference on the Hamilton Anxiety Rating Scale of 1.39 points $(P=0.006)$.

Quality of life is significantly decreased in patients with CVD suffering from depression, and improvement or restoration of quality of life is an important aspect of disease management. In the current study, agomelatine was associated with an improvement in both physical and mental quality-of-life components, which occurred early in the study and continued to improve until study completion.

Although this is the first observational study of agomelatine in an exclusively CVD population, several other observational studies have examined the treatment effects and tolerability of the drug for depressive symptoms in a broad range of patients in clinical practice. In the large CHRONOS observational study conducted in Russia, patients 
Table 5 Physical measures at baseline (week 0) over the 12-week treatment period

\begin{tabular}{llllll}
\hline Parameter & Week 0 & Week 3 & Week 6 & Week I2 & P-value \\
\hline Body weight $(\mathrm{kg})$ & $79.3 \pm 14.7$ & $79.1 \pm 7.8$ & $78.6 \pm 11.8$ & $78.2 \pm 13.7$ & $<0.000$ I \\
Heart rate $(\mathrm{bpm})$ & $75.9 \pm 10.6$ & $70.8 \pm 7.8$ & $68.2 \pm 6.5$ & $67.5 \pm 6.5$ & $<0.000$ I \\
SBP $(\mathrm{mmHg})$ & $139.6 \pm 17.5$ & $130.5 \pm 12.1$ & $126.7 \pm 10.5$ & $124.7 \pm 9.4$ & $<0.000$ I \\
DBP $(\mathrm{mmHg})$ & $85.5 \pm 10.2$ & $80.9 \pm 7.6$ & $78.9 \pm 7.2$ & $78.0 \pm 6.5$ & $<0.000$ I \\
\hline
\end{tabular}

Notes: There were 876 patients included in this analysis. All values are presented as mean \pm standard deviation.

Abbreviations: bpm, beats per minute; SBP, systolic blood pressure; DBP, diastolic blood pressure.

demonstrated statistically significant changes in the HAM-D during treatment. ${ }^{23}$ A German observational study, which included severely depressed patients and elderly patients with comorbid conditions, including CVD, also reported improvements throughout the study. ${ }^{21}$

The efficacy of agomelatine in depression is thought to be due to its pharmacological profile, acting as both an agonist to melatonergic receptors and an antagonist to $5 \mathrm{HT} 2 \mathrm{C}$ receptors. These receptors act in synergy to increase dopaminergic and noradrenergic neurotransmission, and there is a selective release of both neurotransmitters in the prefrontal cortex..$^{31,32}$ Furthermore, it has no effect on extracellular serotonin in the brain, and thus, there is no dampening effect of serotonin in the release of dopamine, as may occur with the SSRIs and serotonin-norepinephrine reuptake inhibitors (SNRIs). ${ }^{33,34}$ This potentiation of noradrenaline and dopamine with no effect on serotonin release is consistent with the known clinical actions of agomelatine in depression including improvement of anhedonia and reduction of emotional blunting. This receptor profile is also responsible for the good adverse event profile of agomelatine, which differs from SSRI and SNRI antidepressants, especially with regard to gastrointestinal side effects, headache, sexual dysfunction, and psychomotor agitation. ${ }^{35}$ Even though no head-to-head studies with agomelatine and comparator treatments have been conducted in patients with CVD and

Table 6 Documented liver parameters over the 12-week treatment period

\begin{tabular}{|c|c|c|c|c|c|}
\hline Index & Week 0 (baseline) & Week 3 & Week 6 & Week I2 & $P_{0-12}$-value \\
\hline AST (U/L) & $23.7 \pm 8.6$ & $24.2 \pm 8.4$ & $24.1 \pm 8.5$ & $24.5 \pm 8.9$ & 0.021 \\
\hline \multicolumn{6}{|c|}{ Percentage of patients with an AST value } \\
\hline$\leq \mathrm{ULN}$ & 100 & 100 & 95.85 & 93.75 & \\
\hline$\leq 3$ ULN & 0 & 0 & 4.15 & 6.25 & \\
\hline$>3$ ULN & 0 & 0 & 0 & 0 & \\
\hline ALT (U/L) & $24.6 \pm 10.2$ & $25.0 \pm 9.7$ & $25.2 \pm 9.6$ & $25.4 \pm 9.8$ & 0.018 \\
\hline \multicolumn{6}{|c|}{ Percentage of patients with an ALT value } \\
\hline$\leq \mathrm{ULN}$ & 100 & 100 & 97.15 & 94.31 & \\
\hline$\leq 3$ ULN & 0 & 0 & 2.85 & 5.69 & \\
\hline$>3 U L N$ & 0 & 0 & 0 & 0 & \\
\hline ALP (U/L) & $111.3 \pm 69.8$ & $113.4 \pm 69.0$ & $1 \mid 4.6 \pm 68.1$ & $1 \mid 5.1 \pm 69.1$ & 0.007 \\
\hline \multicolumn{6}{|c|}{ Percentage of patients with an ALP value } \\
\hline$\leq \mathrm{ULN}$ & 99.8 & 99.8 & 99.54 & 99.54 & \\
\hline$\leq 3$ ULN & 0.2 & 0.2 & 0.46 & 0.46 & \\
\hline$>3$ ULN & 0 & 0 & 0 & 0 & \\
\hline GGT (U/L) & $27.8 \pm 12.7$ & $29.2 \pm 13.0$ & $30.3 \pm 13.5$ & $29.6 \pm 12.9$ & 0.012 \\
\hline \multicolumn{6}{|c|}{ Percentage of patients with a GGT value } \\
\hline$\leq \mathrm{ULN}$ & 99.32 & 99.32 & 99.32 & 99.43 & \\
\hline$\leq 3$ ULN & 0.68 & 0.68 & 0.68 & 0.57 & \\
\hline$>3$ ULN & 0 & 0 & 0 & 0 & \\
\hline TB $(\mu \mathrm{mol} / \mathrm{L})$ & $14.2 \pm 4.5$ & $14.7 \pm 4 . \mid$ & $15.0 \pm 4.0$ & $15.0 \pm 4.2$ & $<0.0001$ \\
\hline \multicolumn{6}{|c|}{ Percentage of patients with a TB value } \\
\hline$\leq \mathrm{ULN}$ & 100 & 100 & 100 & 98.97 & \\
\hline$\leq 3$ ULN & 0 & 0 & 0 & 1.03 & \\
\hline$>3$ ULN & 0 & 0 & 0 & 0 & \\
\hline
\end{tabular}

Note: All values are presented as mean \pm standard deviation or percentage of patients.

Abbreviations: AST, aspartate aminotransferase; ALT, alanine aminotransferase; ALP, alkaline phosphatase; GGT, gamma-glutamyl transpeptidase; TB, total bilirubin; ULN, upper limit of normal. 
depression, agomelatine may prove particularly beneficial in these patients because of its favorable CVD safety profile. In addition to efficacy, the side-effect profile of antidepressants and their potential for significant drug interactions is important in patients with CVD as they are likely to be suffering from other comorbidities and taking a number of concomitant medications. In this study, agomelatine was well tolerated, the side effects being those already documented in the Summary of Product Characteristics for agomelatine. There were no cases of liver function parameters exceeding threefold the ULN during the study.

The observed changes in somatic symptom disorders suggest that there was a positive effect of combining agomelatine and a non-psychotropic agent (cardiotropic) that was not accompanied by the development or potentiation of significant adverse events. The improvements in heart rate, and systolic and diastolic blood pressure values could have been due to an effective cardiotropic therapy, or the result of an effective combination of therapies including agomelatine. The ability of agomelatine to normalize patients' mental state may have resulted in a decrease in distressing thoughts, feelings, and behaviors regarding their somatic symptoms, including the frequency and severity of exacerbations of somatic symptoms.

Agomelatine therapy was associated with a decrease in body weight after 12 weeks, but this did not exceed $5 \%$ of body weight at baseline. While the weight loss is unlikely to have been related to the pharmacological properties of agomelatine, it may have been a consequence of the improvements in many of the somatic symptom disorders that were assessed in this study. As such, this is an important finding as weight loss is often considered a positive step in patients with CVD.

A number of antidepressants have unwanted cardiovascular effects, and when treating depression in CVD, the choice of treatment requires a careful balance of safety and efficacy. For example, hypertension can be significant with venlafaxine ${ }^{36}$ and monoamine oxidase inhibitors. Other treatments have been associated with dose-dependent effects on repolarization interval, characterized by lengthening of the QT interval on an electrocardiogram, a marker of arrhythmic risk. ${ }^{37}$ In a pharmacovigilance study, using health records from 38,397 patients treated with antidepressants, statistically significant evidence of modest QT prolongation was identified for the tricyclic antidepressant amitriptyline, as well as for citalopram and escitalopram. ${ }^{38}$ In contrast, agomelatine up to $400 \mathrm{mg}$ has been shown to have no effect on the QTc interval in healthy volunteers. ${ }^{39}$
Some antidepressant agents with demonstrated efficacy in major depressive disorder may be less effective in patients with certain cardiac conditions. For example, both the SADHART-CHF study with sertraline ${ }^{13}$ and the recently published MOOD-HF study (Effects of Selective Serotonin Re-Uptake Inhibition on Morbidity, Mortality, and Mood in Depressed Heart Failure Patients) with escitalopram ${ }^{40}$ showed that while the drugs were safe, they did not provide a greater reduction in depressive symptoms than placebo or have a greater impact on cardiovascular events or survival. This was despite the fact that MOOD-HF followed patients for over 18 months. ${ }^{40}$ These findings led both sets of study authors to suggest that the pathophysiology of depression in patients with heart failure may have unique characteristics that may be less responsive to some antidepressant therapies. In the current observational study, agomelatine was effective in patients presenting with a range of CVDs including hypertension, angina, myocardial infarction, atherosclerosis, rhythm and conduction disturbances, as well as chronic heart failure.

This study was subject to the inherent limitations of observational studies including susceptibility to selection bias and confounding. In addition, the number of patients per investigator was small, which could overemphasize the significance of the results. The lack of a control group also limits the conclusions that can be drawn from the study.

Real-world observational studies nevertheless remain valuable for evaluating efficacy and safety of a treatment in a broad range of patients who more closely reflect routine clinical practice than those found in the restricted populations of randomized controlled trials. This also ensures that drug safety is monitored in a broad population of patients.

\section{Conclusion}

Depression and CVD are both widespread among the general population and often occur simultaneously in the same individual. The results of this multicenter, observational study show that agomelatine provides a statistically significant improvement in depressive symptoms, anxiety, and hypochondria in depressed patients with a range of CVDs and demonstrates good tolerability. Quality of life was significantly improved, and the majority of patients and cardiologists reported agomelatine efficacy as good to excellent. The results of this observational study suggest that agomelatine is an effective and safe choice for the treatment of depression in patients with CVD.

\section{Acknowledgments}

The author acknowledges the efforts of Khristina Rogozskaya (Samara Regional Clinical Cardiological Dispensary, 
Samara), Dr Anna Porfirieva (City Hospital \#15, Saint Petersburg), Dr Nikolay Dovgany (Ekaterinburg Regional Clinical Hospital \#1, Ekaterinburg), Dr Elena Zhovtilinova (Orenburg Regional Clinical Hospital, Orenburg), Dr Kirill Soldatov (Kuzbass Cardiology Center, Kemerovo), Dr Vyacheslav Trunov (Regional Clinical Cardiological Dispensary, Tver), Dr Leyla Fachikova (City Clinical Hospital \#7, Kazan), Dr Vasiliy Yanushko (City Clinical Hospital \#5, Nizhny Novgorod), Dr Olyga Ivanova (Ryazan City Hospital \#4, Ryazan), Dr Yulia Samshitova (Kursk Regional Clinical Hospital, Kursk), Dr Anna Trol (City Hospital \#1, Voronezh), Dr Julia Borisova (Diagnostic and Treatment Center, Saratov), Dr Fedor Ovchinkin (Novosibirsk Regional Clinical Hospital, Novosibirsk), Dr Boris Brun (City Hospital \#1, Irkutsk), Dr Grigoriy Kuskov (Municipal Cardiologic Polyclinic, Khabarovsk), Dr Inna Promes (Perm Regional Clinical Hospital, Perm), and Dr Irina Zamurzina (Regional Clinical Hospital, Krasnoyarsk) for coordination of the study. There was no funding source for the study. English language editing was provided by a freelance medical writer and funded by Servier.

\section{Disclosure}

The author reports no conflicts of interest in this work.

\section{References}

1. Frasure-Smith N, Lespérance F. Depression and coronary artery disease. Herz. 2006;31 Suppl 3:64-68.

2. Egede LE. Major depression in individuals with chronic medical disorders: prevalence, correlates and association with health resource utilization, lost productivity and functional disability. Gen Hosp Psychiatry. 2007;29(5):409-416.

3. American Psychiatric Association. Diagnostic and Statistical Manual of Mental Disorders. Washington, DC: American Psychiatric Association; 2000.

4. Kessler RC, Bromet EJ. The epidemiology of depression across cultures. Annu Rev Public Health. 2013;34:119-138.

5. Thombs BD, Bass EB, Ford DE. Prevalence of depression in survivors of acute myocardial infarction. J Gen Intern Med. 2006;21(1):30-38.

6. Rutledge T, Reis VA, Linke SE, Greenberg BH, Mills PJ. Depression in heart failure: a meta-analytic review of prevalence, intervention effects, and associations with clinical outcomes. J Am Coll Cardiol. 2006; 48(8):1527-1537.

7. Nicholson A, Kuper H, Hemingway H. Depression as an aetiologic and prognostic factor in coronary heart disease: a meta-analysis of 6362 events among 146538 participants in 54 observational studies. Eur Heart J. 2006;27(23):2763-2774.

8. Sullivan MD, Levy WC, Crane BA, Russo JE, Spertus JA. Usefulness of depression to predict time to combined end point of transplant or death for outpatients with advanced heart failure. Am J Cardiol. 2004;94(12):1577-1580.

9. Hatcher S, Arroll B. Newer antidepressants for the treatment of depression in adults. BMJ. 2012;344:d8300.

10. Krantz DS, Whittaker KS, Francis JL, et al. Psychotropic medication use and risk of adverse cardiovascular events in women with suspected coronary artery disease: outcomes from the Women's Ischemia Syndrome Evaluation (WISE) study. Heart. 2009;95(23): 1901-1906.
11. Lichtman JH, Bigger JT Jr, Blumenthal JA, et al; American Heart Association Prevention Committee of the Council on Cardiovascular Nursing; American Heart Association Council on Clinical Cardiology; American Heart Association Council on Epidemiology and Prevention; American Heart Association Interdisciplinary Council on Quality of Care and Outcomes Research; American Psychiatric Association. Depression and coronary heart disease: recommendations for screening, referral, and treatment: a science advisory from the American Heart Association Prevention Committee of the Council on Cardiovascular Nursing, Council on Clinical Cardiology, Council on Epidemiology and Prevention, and Interdisciplinary Council on Quality of Care and Outcomes Research: endorsed by the American Psychiatric Association. Circulation. 2008;118(17):1768-1775.

12. Pizzi C, Rutjes AW, Costa GM, Fontana F, Mezzetti A, Manzoli L. Meta-analysis of selective serotonin reuptake inhibitors in patients with depression and coronary heart disease. Am J Cardiol. 2011; 107(7):972-979.

13. O'Connor CM, Jiang W, Kuchibhatla M, et al; SADHART-CHF Investigators. Safety and efficacy of sertraline for depression in patients with heart failure: results of the SADHART-CHF (Sertraline Against Depression and Heart Disease in Chronic Heart Failure) trial. $J$ Am Coll Cardiol. 2010;56(9):692-699.

14. Hansen RA, Khodneva Y, Glasser SP, Qian J, Redmond N, Safford MM. Antidepressant medication use and its association with cardiovascular disease and all-cause mortality in the Reasons for Geographic and Racial Differences in Stroke (REGARDS) Study. Ann Pharmacother. 2016; 50(4):253-261.

15. Castro VM, Gallagher PJ, Clements CC, et al. Incident user cohort study of risk for gastrointestinal bleed and stroke in individuals with major depressive disorder treated with antidepressants. BMJ Open. 2012; 2(2): 000544.

16. Smoller JW, Allison M, Cochrane BB, et al. Antidepressant use and risk of incident cardiovascular morbidity and mortality among postmenopausal women in the Women's Health Initiative study. Arch Intern Med. 2009;169(22):2128-2139.

17. Coupland C, Hill T, Morriss R, Moore M, Arthur A, Hippisley-Cox J. Antidepressant use and risk of cardiovascular outcomes in people aged 20 to 64: cohort study using primary care database. BMJ. 2016; 352:i1350.

18. Lespérance F, Frasure-Smith N. Depression in patients with cardiac disease: a practical review. J Psychosom Res. 2000;48(4-5):379-391.

19. Taylor D, Sparshatt A, Varma S, Olofinjana O. Antidepressant efficacy of agomelatine: meta-analysis of published and unpublished studies. BMJ. 2014;348:g1888.

20. Goodwin GM, Emsley R, Rembry S, Rouillon F; Agomelatine Study Group. Agomelatine prevents relapse in patients with major depressive disorder without evidence of a discontinuation syndrome: a 24-week randomized, double-blind, placebo-controlled trial. J Clin Psychiatry. 2009;70(8):1128-1137.

21. Laux G; VIVALDI Study Group. The antidepressant agomelatine in daily practice: results of the non-interventional study VIVALDI. Pharmacopsychiatry. 2012;45(7):284-291.

22. Gorwood P, Bayle F, Vaiva G, Courtet P, Corruble E, Llorca PM. Is it worth assessing progress as early as week 2 to adapt antidepressive treatment strategy? Results from a study on agomelatine and a global meta-analysis. Eur Psychiatry. 2013;28(6):362-371.

23. Ivanov SV, Samushiya MA. Agomelatine in the treatment of depressive disorders in clinical practice: multicenter observational CHRONOS study. Neuropsychiatr Dis Treat. 2014;10:631-639.

24. Martinotti G, Sepede G, Gambi F, et al. Agomelatine versus venlafaxine $\mathrm{XR}$ in the treatment of anhedonia in major depressive disorder: a pilot study. J Clin Psychopharmacol. 2012;32(4):487-491.

25. Llorca PM. The antidepressant agomelatine improves the quality of life of depressed patients: implications for remission. J Psychopharmacol. 2010;24(2 Suppl):21-26.

26. Guaiana G, Gupta S, Chiodo D, Davies SJ, Haederle K, Koesters M. Agomelatine versus other antidepressive agents for major depression. Cochrane Database Syst Rev. 2013;(12):CD008851. 
27. McConnell S, Jacka FN, Williams LJ, Dodd S, Berk M. The relationship between depression and cardiovascular disease. Int J Psychiatry Clin Pract. 2005;9(3):157-167.

28. Goodwin GM, Rouillon F, Emsley R. Long-term treatment with agomelatine: prevention of relapse in patients with major depressive disorder over 10 months. Eur Neuropsychopharmacol. 2008; 18(Suppl 4):S338-S339.

29. Demyttenaere K, Corruble E, Hale A, Quera-Salva MA, PicarelBlanchot F, Kapser S. A pooled analysis of six month comparative efficacy and tolerability in four randomized clinical trials: agomelatine versus escitalopram, fluoxetine, and sertraline. CNS Spectr. 2013; 18(3):163-170.

30. Stein DJ, Picarel-Blanchot F, Kennedy SH. Efficacy of the novel antidepressant agomelatine for anxiety symptoms in major depression. Hum Psychopharmacol. 2013;28(2):151-159.

31. Stahl SM. Mechanism of action of agomelatine: a novel antidepressant exploiting synergy between monoaminergic and melatonergic properties. CNS Spectr. 2014;19(3):207-212

32. Racagni G, Riva MA, Molteni R, et al. Mode of action of agomelatine: synergy between melatonergic and 5-HT(2C) receptors. World J Biol Psychiatry. 2011;12(8):574-587.

33. Millan MJ, Gobert A, Lejeune F, et al. The novel melatonin agonist agomelatine (S20098) is an antagonist at 5-hydroxytryptamine2C receptors, blockade of which enhances the activity of frontocortical dopaminergic and adrenergic pathways. J Pharmacol Exp Ther. 2003;306(3):954-964.

34. Millan MJ, Brocco M, Gobert A, Dekeyne A. Anxiolytic properties of agomelatine, an antidepressant with melatoninergic and serotonergic properties: role of 5-HT2C receptor blockade. Psychopharmacology (Berl). 2005;177(4):448-458.
35. Hickie IB, Rogers NL. Novel melatonin-based therapies: potential advances in the treatment of major depression. Lancet. 2011;378(9791): 621-631.

36. Thase ME. Effects of venlafaxine on blood pressure: a meta-analysis of original data from 3744 depressed patients. J Clin Psychiatry. 1998; 59(10):502-508

37. Teply RM, Packard KA, White ND, Hilleman DE, DiNicolantonio JJ. Treatment of depression in patients with concomitant cardiac disease. Prog Cardiovasc Dis. 2016;58(5):514-528.

38. Castro VM, Clements CC, Murphy SN, et al. QT interval and antidepressant use: a cross sectional study of electronic health records. BMJ. 2013;346:f288.

39. Donazzolo Y, Latreille M, Caillaud MA, Mocaer E, Seguin L. Evaluation of the effects of therapeutic and supratherapeutic doses of agomelatine on the QT/QTc interval: a phase I, randomized, double-blind, placebo-controlled and positive-controlled, crossover thorough QT/QTc study conducted in healthy volunteers. J Cardiovasc Pharmacol. 2014;64(5):440-451.

40. Angermann CE, Gelbrich G, Störk S, et al; MOOD-HF Study Investigators and Committee Members. Effect of escitalopram on all-cause mortality and hospitalization in patients with heart failure and depression: the MOOD-HF randomized clinical trial. JAMA. 2016;315(24):2683-2693.
Neuropsychiatric Disease and Treatment

\section{Publish your work in this journal}

Neuropsychiatric Disease and Treatment is an international, peerreviewed journal of clinical therapeutics and pharmacology focusing on concise rapid reporting of clinical or pre-clinical studies on a range of neuropsychiatric and neurological disorders. This journal is indexed on PubMed Central, the 'PsycINFO' database and CAS,

\section{Dovepress}

and is the official journal of The International Neuropsychiatric Association (INA). The manuscript management system is completely online and includes a very quick and fair peer-review system, which is all easy to use. Visit http://www.dovepress.com/testimonials.php to read real quotes from published authors. 